\title{
Les douleurs induites : un ouvrage de l'institut UPSA de la douleur, mis à jour et complété
}

\author{
Induced pain: a UPSA (Union for Applied Pharmacological Science) \\ Institute for Pain project, updated and completed
}

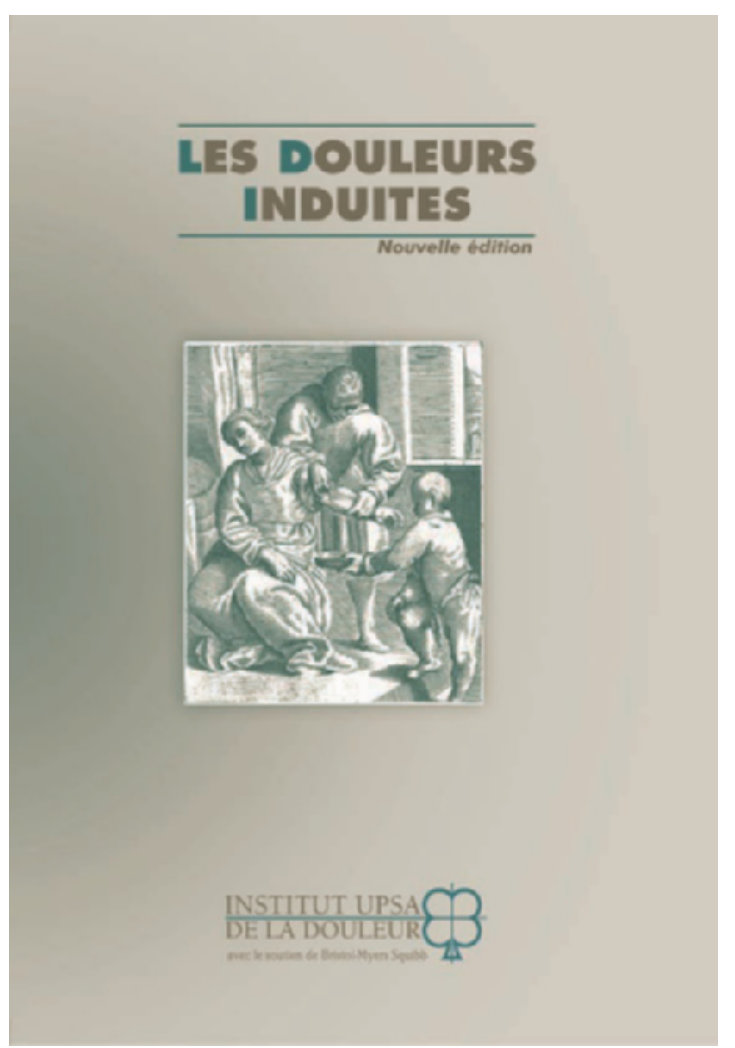

Paru en 2005, Les douleurs induites était le premier ouvrage réalisé sur le sujet. Parce que l'accès à une information de qualité est important mais que l'accès à une information à jour est primordial, l'institut UPSA de la douleur procède régulièrement à l'actualisation de ses données.

Après quelques années d'évolution dans ce domaine, le moment était venu pour l'institut de mettre à jour cet ouvrage.

Disponible sur le site Internet de l'institut UPSA de la douleur (www.institut-upsa-douleur.org), cette nouvelle édition, enrichie et coordonnée par Stéphane Donnadieu et Jacques Wrobel, donne la parole à Frédéric Aubrun, Jean-Marc Benhaiem, Jean-Jacques Eledjman, Dominique Fletcher,
Pascale Fouassier, Francine Hisrzowski, Isabelle Jubin, Virginie Lvovschi, Evelyne Malaquin-Pavan, Paul Pionchon, Christine Ricard, Bruno Riou, Annick Sachet, Esther Soyeux, Eric Viel et Claire Vulser-Cristofini.

Les auteurs apportent leur contribution quant à la mise à jour des données et abordent de nouveaux sujets de réflexion sur les douleurs induites et leur prévention.

\section{Qu'est-ce que les douleurs induites ? Quelles en sont les causes ? Comment les prévenir ? Quelles sont les mesures à suivre ?}

Ainsi, Stéphane Donnadieu déclare que « Les douleurs induites, cette dénomination étant celle à retenir pour les dépister, les évaluer, les réduire, sont des douleurs indues, causées par la réalisation d'actes à visées thérapeutiques ou diagnostiques. Ces actes étant toujours prescrits et réalisés par des soignants (chirurgien, dentiste, infirmière, médecin), l'implication de ceux-ci est évidente dans leur prévention et leur soulagement. \ et apporte des pistes de réflexion pour les soignants : « La banalité, la fréquence des gestes les provoquant font que les soignants les côtoient quotidiennement sans penser à les évaluer ni à informer de leur survenue. Certains mots prononcés par les soignants peuvent même être source de douleur, et cela, en dehors de toute psychopathologie. »"

Causes, traitement ou encore prévention, la douleur induite est ici expliquée, analysée et envisagée sous de nouvelles perspectives et en fonction de mises en situations diverses comme aux urgences, sur un plateau de technique interventionnelle, en période postopératoire, dans un service d'hospitalisation, en médecine générale ou encore en odontostomatologie, soit selon des types particuliers de patients tels que les personnes âgées et les enfants. De plus, deux nouveaux chapitres : « Douleurs induites et hypnose » ainsi que « Épidémiologie, mécanisme d'action et facteurs prédictifs des douleurs induites » viennent enrichir cet ouvrage. 